\title{
Effects of the density dependence of nuclear symmetry energy on properties of superheavy nuclei
}

\author{
Wei-Zhou Jiang \\ 1 Department of Physics, Southeast University, Nanjing 211189, China \\ 2 National Laboratory of Heavy Ion Accelerator, Lanzhou 730000, China

\begin{abstract}
Effects of the density dependence of the nuclear symmetry energy on ground-state properties of of the symmetry energy plays an important role in the empirical shift [Phys. Rev. C 67, 024309
\end{abstract} \\ superheavy nuclei are studied in the relativistic mean-field theory. It is found that the softening \\ (2003)] of spherical orbitals in superheavy nuclei. The calculation based on the relativistic mean- \\ field models NL3 and FSUGold supports the double shell closure in ${ }^{292} 120$ with the softening of \\ the symmetry energy. In addition, the significant effect of the density dependence of the symmetry \\ energy on the neutron skin thickness in superheavy nuclei are investigated.
}

PACS numbers: 21.10.Dr, 21.60.Jz, 27.90.+b

\section{INTRODUCTION}

The persistent interest in the synthesis of superheavy nuclei (SHN) acquires refreshment due to recent progresses [1-14]. This is a hot field where people often expect the next new superheavy element (SHE) that can be synthesized in the laboratory. Indeed, since the cross section of the SHE synthesis is very small, it is much more difficult to synthesize the heavier and heavier SHE [2]. For instance, the cross section of the cold fusion reduces almost exponentially with the increase of the nuclear charge in the superheavy region. One of important factors that affects the synthesis is the shell closure in superheavy nuclei. However, predictions turned out to be quite divisive for various theoretical approaches. For instance, the microscopic-macroscopic model predicts the the double shell closure at $(\mathrm{Z}=114, \mathrm{~N}=184)[15]$; various nonrelativistic models with Skyrme forces can predict different double shell closures at $(\mathrm{Z}=114, \mathrm{~N}=184)[16],(120,172)[16-18]$ or $(126,184)[16,17,19]$, while most relativistic mean-field (RMF) models incline the double shell closure at $(120,172)[16$, $17,20]$. In general, the diversity of predictions on the shell closure in the superheavy region is associated with various single-particle properties near the Fermi surface.

In the recent decade, the extraction of the constraint on the density dependence of the symmetry energy has been another hot spot in nuclear physics due to the availability of high-quality radioactive beams. The density dependence of the symmetry energy plays important roles in understanding many important issues in astrophysics, see, e.g., Refs. [21-23], properties of protonor neutron-rich nuclei and the reaction dynamics of heavy-ion collisions, see, e.g., Refs. [24-27]. However, the density dependence of the symmetry energy is still poorly known especially at high densities [27]. Recent extraction of the neutron skin thickness of ${ }^{208} \mathrm{~Pb}$ from collective flow data 
of heavy ion collisions [28-30] exhibited the softening tendency of the symmetry energy. Since the density dependence of the symmetry energy can reflect the surface property of the isovector potential, the effect on the single-particle property and characteristic of the shell closure in SHN may be induced by the softening of the symmetry energy. Moreover, it was found that the existence of the central depression is important for the double shell closure in ${ }^{292} 120$ [16, 31, 32]. In presence of the central depression, the sensitivity of the properties of SHN to various density dependences of the symmetry energy can be affected. Though the properties of SHN have been explored in a great number of works [15-20, 31-45], the investigation on the symmetry energy dependent effect is scarce. Thus, it is the aim of this work to investigate the effect of the softening of the symmetry energy on ground-state properties of SHN, especially the shell closure.

In the past, the isoscalar-isovector coupling was first introduced in RMF models to mimic various density dependences of the symmetry energy in Ref. [22], and its effects on the properties of finite nuclei, nuclear matter and neutron stars have extensively been investigated in the literature [22, $24,25,46-50]$. It is an economic way to simulate various density dependences of the symmetry energy with the inclusion of the isoscalar-isovector coupling in RMF models. In addition, the RMF theory is successful in describing the properties of nuclei from proton drip line to neutron drip line, since it can provide a dynamic description for the spin-orbit interaction, e.g., see reviews in Refs. [51-53]. In this work, we thus perform the investigation with RMF models. The paper is arranged in the following. In section II, the brief formulas are given for RMF models. The results and discussions are presented in section III. At last, a summary is given in section IV.

\section{A BRIEF FORMALISM}

The relativistic lagrangian can be written as:

$$
\begin{aligned}
\mathcal{L}= & \bar{\psi}\left[i \gamma_{\mu} \partial^{\mu}-M_{N}+g_{\sigma} \sigma-g_{\omega} \gamma_{\mu} \omega^{\mu}-g_{\rho} \gamma_{\mu} \tau_{3} b_{0}^{\mu}-e \frac{1}{2}\left(1+\tau_{3}\right) \gamma_{\mu} A^{\mu}\right] \psi \\
& -\frac{1}{4} F_{\mu \nu} F^{\mu \nu}+\frac{1}{2} m_{\omega}^{2} \omega_{\mu} \omega^{\mu}-\frac{1}{4} B_{\mu \nu} B^{\mu \nu}+\frac{1}{2} m_{\rho}^{2} b_{0 \mu} b_{0}^{\mu}-\frac{1}{4} A_{\mu \nu} A^{\mu \nu} \\
& +\frac{1}{2}\left(\partial_{\mu} \sigma \partial^{\mu} \sigma-m_{\sigma}^{2} \sigma^{2}\right)+U\left(\sigma, \omega^{\mu}, b_{0}^{\mu}\right)
\end{aligned}
$$

where $\psi, \sigma, \omega$, and $b_{0}$ are the fields of the nucleon, scalar, vector, and neutral isovector-vector, with their masses $M_{N}, m_{\sigma}, m_{\omega}$, and $m_{\rho}$, respectively. $A_{\mu}$ is the photon field. $g_{i}(i=\sigma, \omega, \rho)$ are the corresponding meson-nucleon couplings. $F_{\mu \nu}, B_{\mu \nu}$ and $A_{\mu \nu}$ are the strength tensors of $\omega$ and $\rho$ mesons, and photon, respectively

$$
F_{\mu \nu}=\partial_{\mu} \omega_{\nu}-\partial_{\nu} \omega_{\mu}, B_{\mu \nu}=\partial_{\mu} b_{0 \nu}-\partial_{\nu} b_{0 \mu}, A_{\mu \nu}=\partial_{\mu} A_{\nu}-\partial_{\nu} A_{\mu}
$$


The self-interacting terms of $\sigma, \omega$ mesons and the isoscalar-isovector coupling are given generally as

$$
\begin{aligned}
U\left(\sigma, \omega^{\mu}, b_{0}^{\mu}\right)= & -\frac{1}{3} g_{2} \sigma^{3}-\frac{1}{4} g_{3} \sigma^{4}+\frac{1}{4} c_{3}\left(\omega_{\mu} \omega^{\mu}\right)^{2} \\
& +4 g_{\rho}^{2} g_{\omega}^{2} \Lambda_{\mathrm{v}} \omega_{\mu} \omega^{\mu} b_{0 \mu} b_{0}^{\mu}
\end{aligned}
$$

Here, the isoscalar-isovector coupling term is introduced to modify the density dependence of the symmetry energy.

Using the Euler-Lagrangian equation, the equations of motion for nucleons and mesons can be obtained. In the RMF approximation, the mesons are approximated by their classic fields with quantum motion neglected. The Dirac equation in RMF is written as

$$
\left[-i \alpha \cdot \nabla+\beta M_{N}^{*}+g_{\omega} \omega_{0}(r)+g_{\rho} \tau_{3} b_{0}(r)+e \frac{1}{2}\left(1+\tau_{3}\right) A_{0}(r)\right] \psi_{\alpha}(r)=E_{\alpha} \psi_{\alpha}
$$

with $M_{N}^{*}=M_{N}-g_{\sigma} \sigma(r)$ and $E_{\alpha}$ the single-particle energy. For simplicity, the isospin subscript for the $\rho$-meson field is omitted hereafter. For the mesons and photon, the equations of motion are given as

$$
\left(\Delta-m_{\phi}^{2}\right) \phi(r)=-s_{\phi}(r)
$$

where for the photon, $m_{\phi}=0$, and

$$
s_{\phi}(r)=\left\{\begin{array}{cl}
g_{\sigma} \rho_{s}(r)-g_{2} \sigma^{2}(r)-g_{3} \sigma^{3}(r), & \sigma, \\
g_{\omega} \rho_{B}(r)-c_{3} \omega_{0}^{3}-8 g_{\omega}^{2} g_{\rho}^{2} \Lambda_{\mathrm{v}} \omega_{0}(r) b_{0}^{2}(r), & \omega, \\
g_{\rho} \rho_{3}(r)-8 g_{\rho}^{2} g_{\omega}^{2} \Lambda_{\mathrm{v}} b_{0}(r) \omega_{0}^{2}(r), & \text { rho }, \\
e \rho_{c}(r), & \text { photon. }
\end{array}\right.
$$

Here $\rho_{s}, \rho_{B}, \rho_{3}$ and $\rho_{c}$ are the scalar, vector, isovector and charge densities, respectively. We see that the fields $b_{0}$ and $\omega_{0}$ can be modified by the isoscalar-isovector coupling. This modification can also affect the spin-orbit potential which is written as

$$
U_{l s}=\frac{1}{2 M_{\epsilon}^{2}} \frac{d}{r d r}\left(V^{\sigma}(r)+V(r)\right) \mathbf{L} \cdot \mathbf{S}
$$

where

$$
\begin{aligned}
M_{\epsilon} & =M_{N}^{*}+E_{\alpha}-V(r) \approx 2 M_{N}-\left(g_{\sigma} \sigma(r)+V(r)\right), \\
V(r) & =g_{\omega} \omega_{0}(r)+g_{\rho} b_{0}(r) t+e\left(\frac{1+t}{2}\right) A_{0},
\end{aligned}
$$

with $t= \pm 1$ for the proton and neutron, respectively. 
The total binding energy is given as

$$
\begin{aligned}
B= & E_{N}+E_{\sigma}+E_{\omega_{0}}+E_{b_{0}}+E_{c}+E_{C M} \\
= & \sum_{\alpha}\left(E_{\alpha}-M_{N}\right)-\frac{1}{2} \int d^{3} r\left[g_{\sigma} \sigma(r) \rho_{s}(r)+\frac{1}{3} g_{2} \sigma^{3}(r)+\frac{1}{2} g_{3} \sigma^{4}(r)\right] \\
& +\frac{1}{2} \int d^{3} r\left[g_{\omega} \omega_{0}(r) \rho_{B}(r)+\frac{c_{3}}{2} \omega_{0}^{4}(r)\right] \\
& +\frac{1}{2} g_{\rho} \int d^{3} r b_{0}(r)\left[\rho_{3}(r)+8 g_{\rho} g_{\omega}^{2} \Lambda_{\mathrm{v}} \omega_{0}^{2}(r) b_{0}(r)\right] \\
& +\frac{1}{2} e \int d^{3} r A_{0}(r) \rho_{c}(r)-\frac{3}{4} 41 A^{1 / 3} .
\end{aligned}
$$

In practical calculations, the BCS pairing interaction is also included using the constant pairing gaps which are obtained from the prescription of Möller and Nix [54]: $\Delta_{n}=4.8 / N^{1 / 3}, \Delta_{p}=$ $4.8 / Z^{1 / 3}$ with $\mathrm{N}$ and $\mathrm{Z}$ the neutron and proton numbers, respectively. This prescription was also used for the SHN in Ref. [43]. The cut-off $82 A^{-1 / 3} \mathrm{MeV}$ above the nucleon chemical potentials is used to normalize the pairing energy [52]. The coupled Dirac and meson equations are solved for spherical nuclei with an iterative procedure. Details in solving the equations can be found in the literature [51-53], and are not reiterated here.

\section{RESULTS AND DISCUSSIONS}

We first study the properties of the SHN with the RMF parameter set NL3 [55] where the isoscalar-isovector coupling is taken into account to mimic various density dependences of the symmetry energy. For comparisons, calculations are also performed with the RMF parameter set FSUGold [48] that features the isoscalar-isovector coupling. In RMF models, the symmetry energy can be written as:

$$
E_{s y m}=\frac{1}{2}\left(\frac{g_{\rho}}{m_{\rho}^{*}}\right)^{2} \rho_{B}+\frac{k_{F}^{2}}{6 E_{F}^{*}}=\frac{1}{2 \delta} g_{\rho} b_{0}+\frac{k_{F}^{2}}{6 E_{F}^{*}}
$$

where $m_{\rho}^{*}$ is the $\rho$-meson effective mass with $m_{\rho}^{*}=\sqrt{m_{\rho}^{2}+8 \Lambda_{\mathrm{v}}\left(g_{\omega} g_{\rho} \omega_{0}\right)^{2}}, \delta$ is the isospin asymmetry with $\delta=\rho_{3} / \rho_{B}$, and $E_{F}^{*}$ is the Fermi energy. The first term is the potential part of the symmetry energy, and the second term is the kinetic part. The modification to the symmetry energy is dictated by the potential part through the isoscalar-isovector coupling. For a given $\Lambda_{\mathrm{v}}$, we follow Ref. [22] to readjust the $\rho N N$ coupling constant $g_{\rho}$ so as to keep the symmetry energy unchanged at $k_{F}=1.15 \mathrm{fm}^{-1}\left(\rho=0.7 \rho_{0}\right)$. As shown in Fig. 1, the symmetry energy is softened by the isoscalar-isovector coupling. With this softening of the symmetry energy, the appreciable reduction of the neutron skin thickness in heavy nuclei can be obtained without compromising the success in reproducing a variety of ground-state properties [22]. Due to the inclusion of the 
isoscalar-isovector coupling, the total binding energy of heavy nuclei changes by a few $\mathrm{MeV}$, and in SHN this change can rise moderately. To reduce the variation of the binding energy in SHN, one may readjust slightly the parameters such as the meson-nucleon coupling constants and mesons. Without priority, here we readjust slightly the $\sigma$ meson mass $m_{\sigma}$. For simplicity, we do not perform the best-fit procedure, and the value of $m_{\sigma}$ is just refitted to the binding energy of ${ }^{208} \mathrm{~Pb}$. The readjusted parameters with various $\Lambda_{\mathrm{v}}$ and properties of ${ }^{208} \mathrm{~Pb}$ are listed in table I. Except for the original parameter sets NL3 and FSUGold, other parameter sets listed in Table I are named according to the value of $\Lambda_{\mathrm{v}}$. Next, we perform calculations for SHN with these parameter sets and examine the sensitivity of ground-state properties of SHN to differences in the symmetry energy.

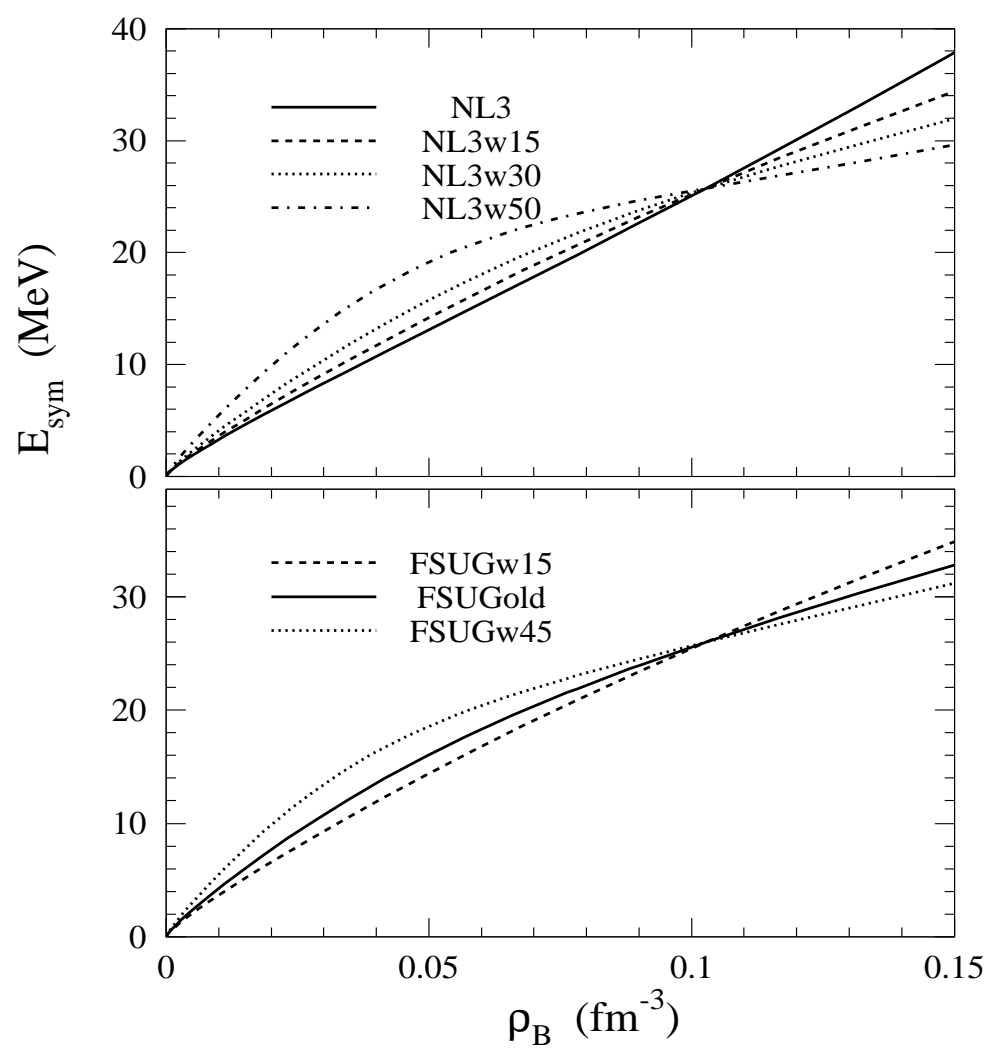

FIG. 1: Density dependence of the symmetry energy with various isoscalar-isovector couplings in NL3 and FSUGold.

In Fig. 2, we plot the single-particle energies for ${ }^{292} 120$ in the NL3 calculations. Results with various isoscalar-isovector couplings are displayed in columns. The large gaps for $\mathrm{N}=172$ and $\mathrm{Z}=120$, as shown in Fig. 2 indicate that the nucleus ${ }^{292} 120$ is doubly magic. It is seen that the shell closure at $\mathrm{N}=172$ and $\mathrm{Z}=120$ undergoes a small but favorable enhancement due to the inclusion of the isoscalar-isovector coupling. As shown in the left panel, the position of $\pi 1 h_{9 / 2}$ relative to that of $\pi 3 s_{1 / 2}$ shifts appreciably with the inclusion of the isoscalar-isovector coupling. For the large $\Lambda_{\mathrm{v}}$, even the level inversion takes place. This shift is in favorably agreement with the one called 
TABLE I: Readjusted parameters in NL3 and FSUGold with ground-state properties of ${ }^{208} \mathrm{~Pb}$. The binding energy per nucleon $(\mathrm{B} / \mathrm{A})$, proton radius $\left(r_{p}\right)$ and neutron skin thickness $\left(r_{p}-r_{n}\right)$ are listed. The slightly modified incompressibility is listed in the last column.

\begin{tabular}{c|ccccccc}
\hline Model & $\Lambda_{\mathrm{v}}$ & $g_{\rho}$ & $m_{\sigma}(\mathrm{MeV})$ & $\mathrm{B} / \mathrm{A}(\mathrm{MeV})$ & $r_{p}(\mathrm{fm})$ & $r_{n}-r_{p}(\mathrm{fm})$ & $\kappa(\mathrm{MeV})$ \\
\hline NL3 & 0.000 & 4.4740 & 508.194 & 7.889 & 5.459 & 0.281 & 271.78 \\
NL3w15 & 0.015 & 4.9652 & 508.240 & 7.890 & 5.465 & 0.238 & 272.25 \\
NL3w30 & 0.030 & 5.6642 & 508.270 & 7.890 & 5.475 & 0.195 & 272.56 \\
NL3w50 & 0.050 & 7.3236 & 508.270 & 7.890 & 5.496 & 0.132 & 272.56 \\
\hline FSUGw15 & 0.015 & 5.0403 & 491.490 & 7.883 & 5.463 & 0.248 & 229.96 \\
FSUGold & 0.030 & 5.8837 & 491.500 & 7.883 & 5.473 & 0.207 & 230.00 \\
FSUGw45 & 0.045 & 7.3695 & 491.480 & 7.883 & 5.488 & 0.158 & 229.92 \\
\hline
\end{tabular}

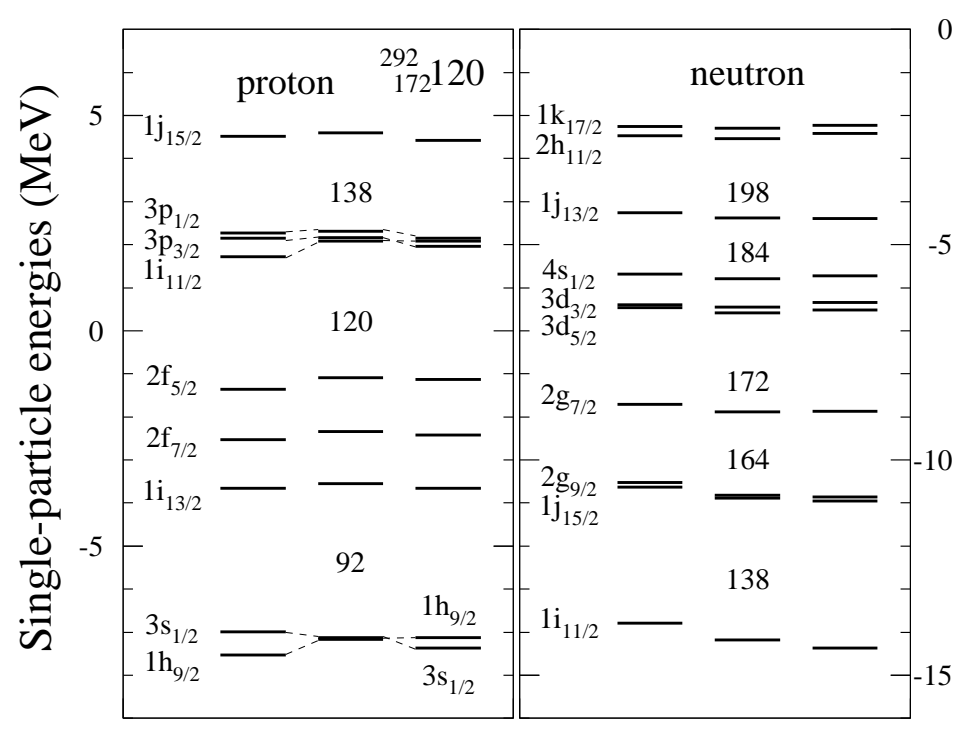

NL3 NL3w30 NL3w50 NL3 NL3w30 NL3w50

FIG. 2: Single-particle energies in ${ }^{292} 120$ with various parameter sets in the NL3 calculations.

as the empirical shift in Refs. [33, 34]. Up to now, there is no direct data of the single-particle energies of SHN, while the so-called empirical shift is obtained by extracting available single-particle energies in deformed nuclei of the $A \sim 250$ region (e.g., ${ }^{249} \mathrm{Bk}$ ) [56-59]. Since several deformed single-particle levels observed in the $A \sim 250$ nuclei emerge from spherical subshells in SHN, the appropriate description of empirical shifts can provide a favorable support for the predictions on properties of SHN, especially the nuclear magicity. With the inclusion of the isoscalar-isovector coupling, the empirical shift between the $\pi 1 h_{9 / 2}$ and $\pi 3 s_{1 / 2}$ can be well reproduced. It is also interesting to see that the low- $j$ levels $\pi 3 p_{3 / 2}$ and $\pi 3 p_{1 / 2}$ can be significantly modified by the isoscalar-isovector coupling. However, its influence on the shell closure at $\mathrm{Z}=120$ remains small. As a result, the shell gap for $\mathrm{Z}=120$ is just weakly affected by the empirical shift. This means 
that these parameter sets including the original NL3 can provide a reliable prediction on the shell closure at $\mathrm{Z}=120$. On the other hand, the empirical shift for $\nu 1 i_{11 / 2}$ [33] in the single-neutron spectrum is not reproduced with the inclusion of the isoscalar-isovector coupling. In Ref. [33], it can be seen that the shell closure at $\mathrm{N}=172$ is just moderately affected by the empirical shift. The present prediction on the large gap for $\mathrm{N}=172$ does not contradict with the early analysis with the empirical shift. Indeed, the relative energies between $\nu 2 g_{9 / 2}, \nu 2 g_{7 / 2}$ and $\nu 3 d_{5 / 2}$, one of which determines the $\mathrm{N}=172$ gap, are almost independent of RMF parametrizations, see Ref. [33] and references therein. The situation of the neutron shell closure at $\mathrm{N}=184$ is less known, since there is no empirical constraint on the $\nu 4 s_{1 / 2}$. However, the $\mathrm{N}=184$ gap is almost independent of the shifts of the interior levels caused by the inclusion of the isoscalar-isovector coupling. Similarly, the $\mathrm{N}=184$ gap would not be much affected even if the empirical shift for $\nu 1 i_{11 / 2}$ is accurately reproduced. In this sense, the occurrence of the shell closure at $\mathrm{N}=184$ seems unlikely.

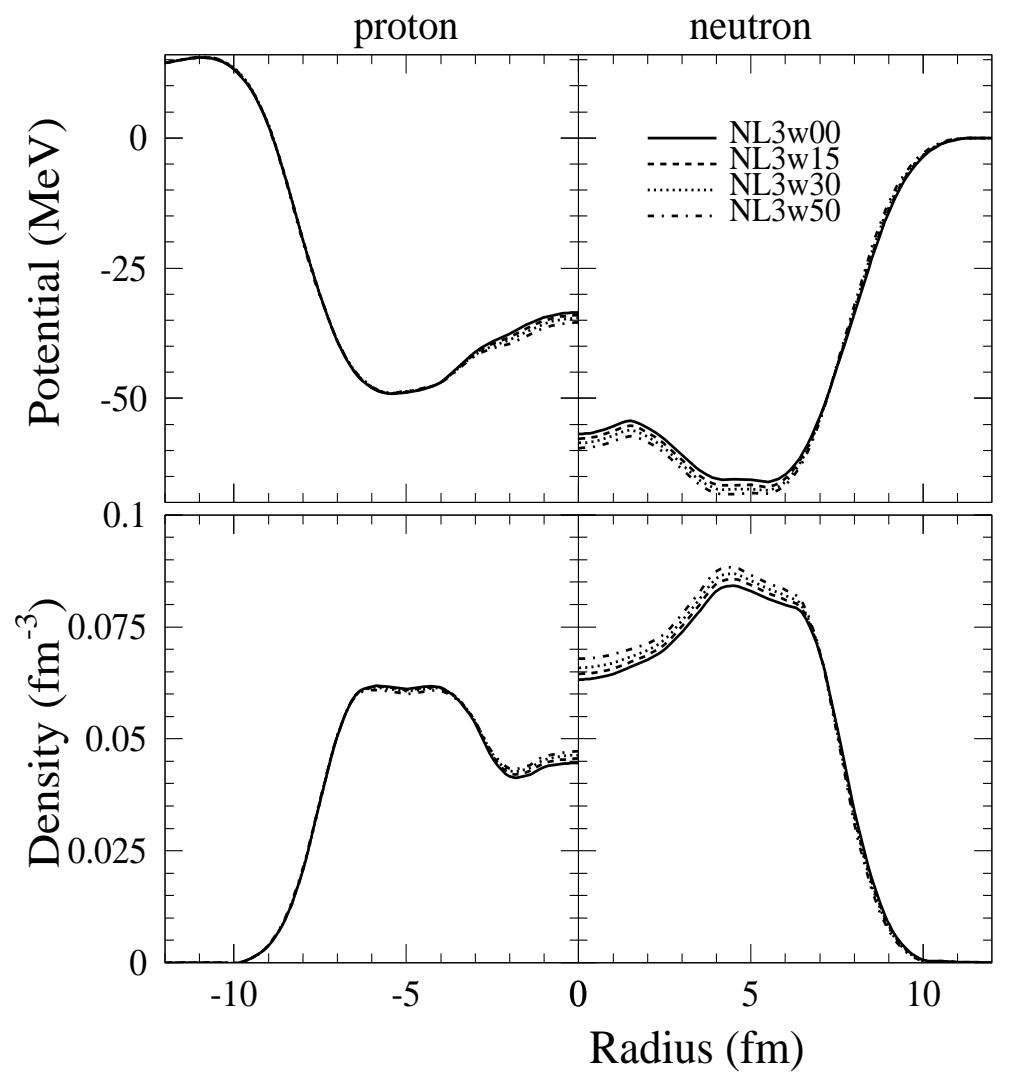

FIG. 3: Nucleon potentials (upper panels) and nucleon density distributions (lower panels) in ${ }^{292} 120$ with various parameter sets in the NL3 calculations. The nucleon potential is defined as $U=V(r)-g_{\sigma} \sigma(r)$, also see Eq.(8).

Now, let's understand underlying factors that intrigue the significant shift in single-particle spectra with the inclusion of the isoscalar-isovector coupling. As shown in Fig. 2, the spin-orbit splitting can be modified by the isoscalar-isovector coupling, and the modification increases moderately with 
the angular momentum. However, the modification to the spin-orbit coupling is just moderate and is not sufficient to cause the empirical shift. Another factor that affects the modification to the single-particle spectrum is the orbit-orbit interaction. The orbit-orbit interaction can generally be given in a form of the centrifugal force, and it reflects the flatness of the nuclear potential. In Fig. 3, the nucleon potentials and density distributions in ${ }^{292} 120$ are plotted. As shown in the upper left panel of Fig. 3, the homogeneity of the proton potential in the central region can be modified by the isoscalar-isovector coupling. This modification can bring about the moderate change in the single-proton levels. Both changes in the orbit-orbit and spin-orbit interactions thus lead to significant empirical shifts of proton levels, observed in ${ }^{292} 120$. Similarly, the shifts in the single-neutron levels can be understood by the modification in the orbit-orbit and spin-orbit interactions caused by the isoscalar-isovector coupling. Though the isoscalar-isovector coupling can affect shifts in the single-particle levels for both protons and neutrons significantly,its modification to the proton or charge radius is much less than that to the neutron radius. This can be seen in Fig. 3 by comparing the modification to the neutron density distribution with that to the proton one.

Furthermore, it is significant to establish a quantitative correlation between the density dependence of the symmetry energy and the relative shift of single-particle energies. It is known that the symmetry energy can be expanded in the vicinity of saturation density in the following form $[60,61]$ :

$$
E_{\text {sym }}\left(\rho_{B}\right)=E_{\text {sym }}\left(\rho_{0}\right)+\frac{L}{3} \frac{\rho_{B}-\rho_{0}}{\rho_{0}}+\frac{\kappa_{s y m}}{18} \frac{\left(\rho_{B}-\rho_{0}\right)^{2}}{\rho_{0}^{2}}+\cdots
$$

where $L$ and $\kappa_{\text {sym }}$ are the slope and curvature of the symmetry energy at saturation density, respectively, defined as

$$
L=\left.3 \rho_{0} \frac{\partial E_{s y m}}{\partial \rho_{B}}\right|_{\rho_{0}}, \quad \kappa_{s y m}=\left.9 \rho_{0}^{2} \frac{\partial^{2} E_{s y m}}{\partial \rho_{B}^{2}}\right|_{\rho_{0}} .
$$

The slope of the symmetry energy defines the symmetry pressure through the relation $p_{\text {sym }}=$ $\rho_{0} L / 3$. In the history, the proper inclusion of the spin-orbit coupling played an important role in giving rise to the correct shape of nuclear potential and hence the ordering of the energy levels. Since the spin-orbit interaction is associated with the surface property subject to the subsaturation density region, it is useful to similarly define the slope and curvature of the symmetry energy at half saturation density, $L^{h}\left(p_{\text {sym }}^{h}\right)$ and $\kappa_{\text {sym }}^{h}$. As seen in Fig. 2, the relative shifts of the orbitals change with respect to the isoscalar-isovector coupling. Since the correlation between these relative shifts and the density dependence of the symmetry energy are similar, we just plot as an example in Fig. 4 the relative shift between $\pi 1 h_{9 / 2}$ and $\pi 3 s_{1 / 2}$ as a function of the symmetry pressure and curvature. 
As shown in Fig. 4, the relative shift of these two levels is approximately linear in the symmetry pressure (at saturation density) and correlates quadratically with the symmetry pressure at half saturation density. A stronger correlation at half saturation density reflects the strong dependence of single-particle energies on the surface property of finite nuclei. In the right panel, it is shown that the relative shift of the two levels is linear in the curvature at half saturation density, which is consistent with the quadric correlation as shown in the middle panel. It was found in the early time for the RMF theory that the proper density dependence of the potential is important for a correct spin-orbit potential and hence the ordering of orbitals, e.g. see [62, 63]. Similarly, it is here interesting to see that the density dependence of the symmetry energy (or of the isovector potential) affects moderately the single-particle energies. In particular, in the present work we can obtain the relative shift between $\pi 1 h_{9 / 2}$ and $\pi 3 s_{1 / 2}$ with the appropriate density dependence of the symmetry energy. The correlations shown in Fig. 4 also exist for other SHN, even if the central depression disappears. Here, the central depression plays a role in enhancing the correlation strength ( e.g. the slope in the linear correlation).

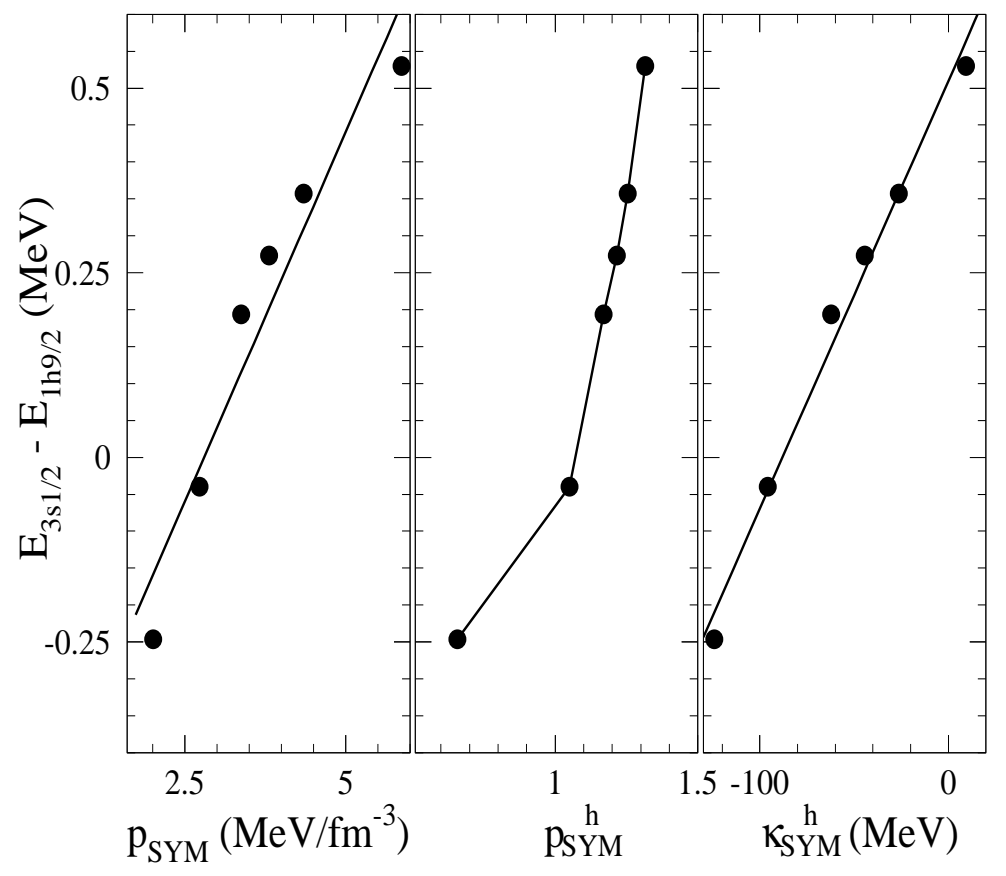

FIG. 4: Relative shift between $\pi 1 h_{9 / 2}$ and $\pi 3 s_{1 / 2}$ as a function of the symmetry pressure and curvature. The line in the left and right panels are obtained with a linear fit. The pressure in the left panel is evaluated at saturation density, while the pressure and curvature in the middle and right panels, respectively, are calculated at half saturation density.

Next, we turn to the discussion on the two-nucleon gaps. Besides the gap in the single-particle spectrum, a direct measure of the shell closure is the appearance of the peak in the two-nucleon 


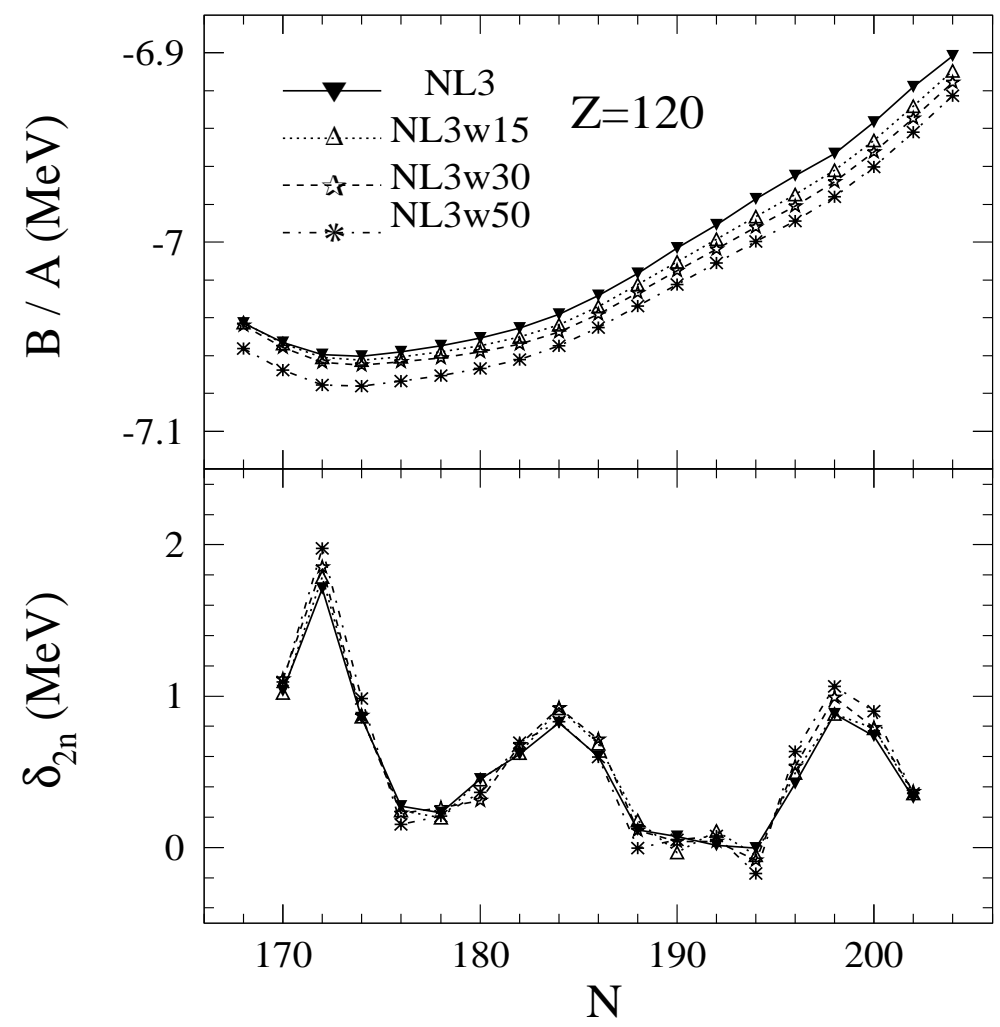

FIG. 5: Binding energies per nucleon of $\mathrm{Z}=120$ isotopes (upper panel) and two-neutron gaps (lower panel) with various parameter sets in the NL3 calculations.

gaps, which are defined as [17]:

$$
\begin{aligned}
& \delta_{2 n}=S_{2 n}(N+2, Z)-S_{2 n}(N, Z)=2 B(N, Z)-B(N-2, Z)-B(N+2, Z), \\
& \delta_{2 p}=S_{2 p}(N, Z+2)-S_{2 p}(N, Z)=2 B(N, Z)-B(N, Z-2)-B(N, Z+2),
\end{aligned}
$$

where $S_{2 n}$ and $S_{2 p}$ are the two-neutron and two-proton separation energies, respectively. The peak of the two-nucleon gap reflects the large change of the two-nucleon separation energy, signaling the shell closure. Moreover, the two-nucleon gap can reflect appropriately the gap size in the single-particle spectrum [16]. In Fig. 5, we plot the binding energy per nucleon (upper panel) and the two-neutron gap (lower panel) for the $\mathrm{Z}=120$ isotopes in the NL3 calculations. As shown in the upper panel of Fig. 5, the difference between binding energies is small for different isoscalarisovector couplings, and this is attributed to the refitting of $m_{\sigma}$. As shown in the lower panel of Fig. 5, the two-neutron gap at $\mathrm{N}=172$ can earn a moderate rise with the inclusion of the isoscalarisovector coupling, which is consistent with the observation of the single-neutron spectrum in Fig. 2. The similar increasing tendency also occurs for the $\mathrm{N}=198$ shell gap. As shown in the lower panel of Fig. 5, the peak of the two-neutron gap occurs at $\mathrm{N}=172,184$ and 198 in the $\mathrm{Z}=120$ isotopes. Comparing to the sharp peak at $\mathrm{N}=172$, peaks at $\mathrm{N}=184$ and 198 becomes much blunt. This 
indicates that the shell gaps at $\mathrm{N}=184$ and 198 are not well developed. Also, we examine the $\delta_{2 n}$ for the $\mathrm{Z}=126$ isotopes, and the case is similar.

As shown in Fig. 5, the two-neutron gap can be affected by the isoscalar-isovector coupling. In some isotopes such as ${ }^{292} 120$ and ${ }^{318} 120$, the two-neutron gap can gain a rise with the inclusion of the isoscalar-isovector coupling. However, the modification to the two-neutron gap caused by the isoscalar-isovector coupling is not governed by the isotopic effect in SHN. For instance, this is clear as comparing the modification to $\delta_{2 n}$ at $\mathrm{N}=172$ with the one at $\mathrm{N}=184$. In fact, the relatively pronounced modification is associated with the specific geometries such as the central depression or enhancement. It was pointed out in Ref. [32] that the central depression in ${ }^{292} 120$ is predominantly from the proton occupation of high-j orbitals, while the neutron central depression results from the strong coupling between protons and neutrons. In presence of the central depression, the change in the neutron potential and density distribution caused by the isoscalar-isovector coupling exhibits a radial inhomogeneity, as shown in right panels of Fig. 3. This inhomogeneity causes consistently modifications to the level shifts and the two-neutron gaps. With the increase of the neutron numbers, the neutron central depression tends to disappear, while the central enhancement appears with more neutrons. The inhomogeneity of the modifications produced in presence of the central enhancement explains the change in the two-neutron gap in more neutron-rich isotopes, as shown in the lower panel of Fig. 5 .

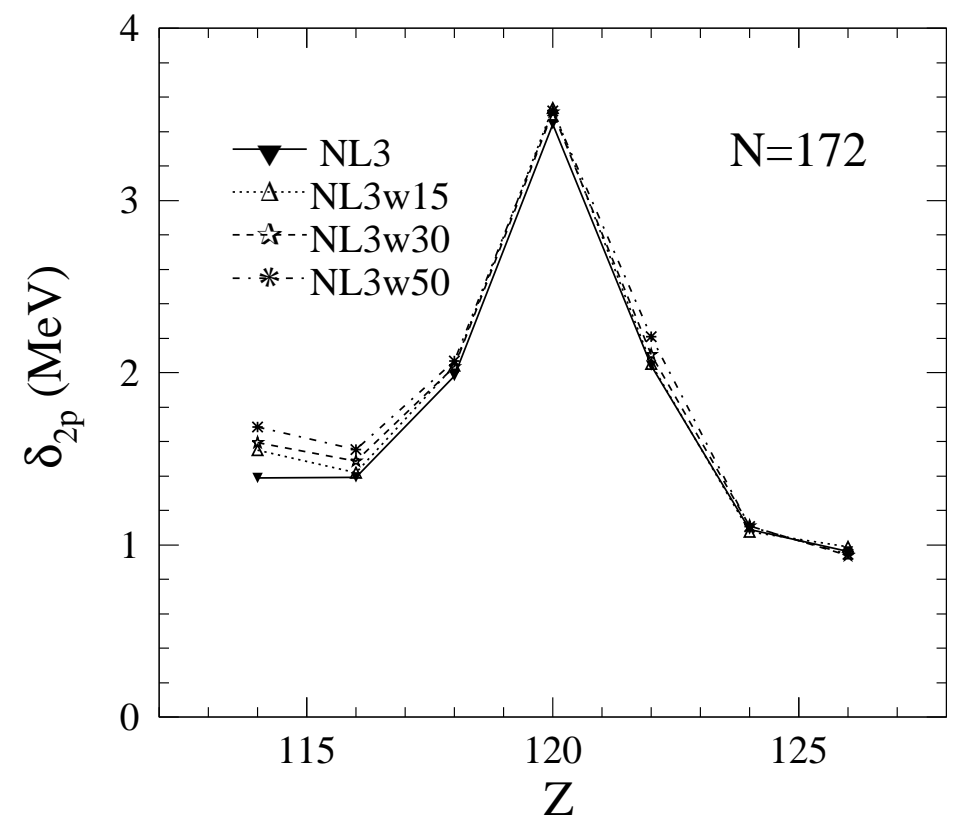

FIG. 6: Two-proton gap $\delta_{2 p}$ for $\mathrm{N}=172$ isotones with various parameter sets in the NL3 calculations.

To examine the proton shell closure, we plot in Fig. 6 the two-proton gap for the $\mathrm{N}=172$ isotones. 
As shown in Fig. 6 , there is only one peak at $\mathrm{Z}=120$. No peak is observed at $\mathrm{Z}=114$ and 126 . The proton shell closure at $Z=126$ was predicted by the Hartree-Fock approach with a variaty of Skyrme interactions $[16,17]$, while the RMF models disincline the appearance of this shell closure. As seen in Fig. 2, though the isoscalar-isovector coupling tends to shift the relative position between $\pi 1 i_{11 / 2}$ and $\pi 3 p$ orbitals, the formation of the $\mathrm{Z}=126$ shell closure does not appear. In RMF models, the $\mathrm{Z}=114$ shell closure was only predicted by parameter sets NL-SH and NL-RA1 due to the relatively large spin-orbit splitting of $2 f$ orbitals $[64,65]$. In NL3, the spin-orbit splitting of $2 f$ orbitals is not large, and though the modification of the isoscalar-isovector coupling to $\delta_{2 p}$ is rather pronounced, as shown in Fig. 6, it is far from sufficient to form the $Z=114$ shell gap. It is necessary to point out that the isotopic effect is prominent for the two-proton gap. For instance, as observed in Fig. 6, the sensitivity of the $\delta_{2 p}$ to the isoscalar-isovector coupling differs clearly for the $\mathrm{N}=172$ isotones with $\mathrm{Z}=114$ and 116 . Indeed, the pronounced isotopic effect exists for the $\mathrm{Z}=120$ shell closure. $\delta_{2 p}=3.5 \mathrm{MeV}$ in ${ }^{292} 120$ reduces to $2.7 \mathrm{MeV}$ in ${ }^{304} 120$. Further, the peak at $\mathrm{Z}=120$ disappears totally in ${ }^{318} 120$. As far as the double shell closure in spherical SHN is concerned, ${ }^{292} 120$ turns out to be the most possible candidate with various parameter sets in the NL3 calculations. This is consistent with the prediction in Refs. [16, 17, 20, 32].

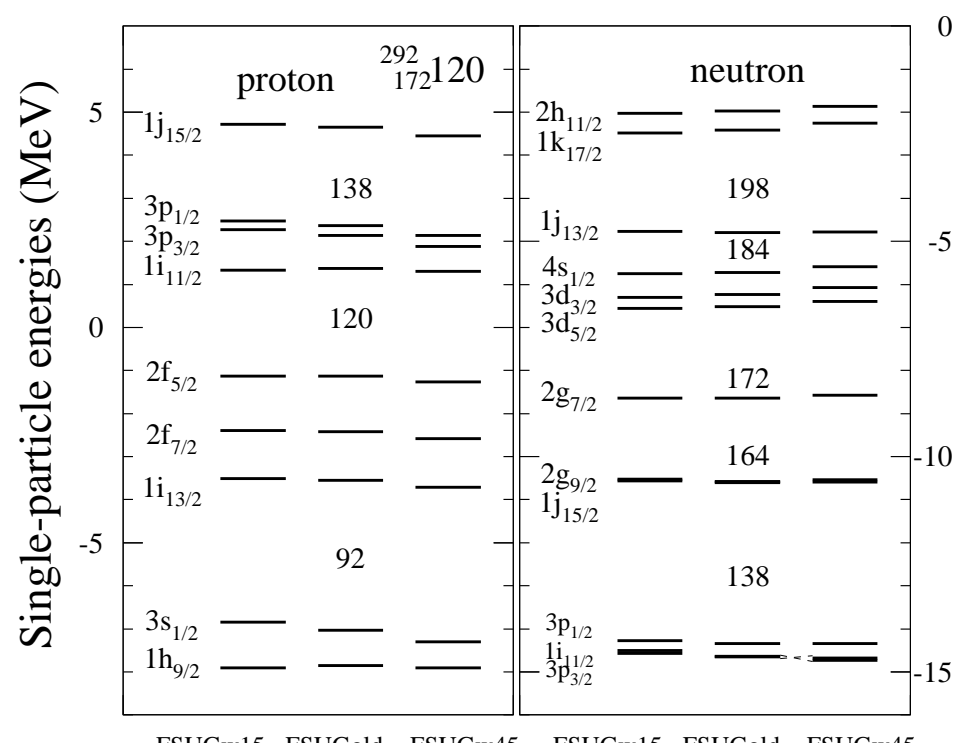

FSUGw15 FSUGold FSUGw45 FSUGw15 FSUGold FSUGw45

FIG. 7: Single-particle energies in ${ }^{292} 120$ with various parameter sets in the FSUGold calculations.

Now, we discuss the results with the FSUGold. In Fig. 7, the single-particle energies for ${ }^{292} 120$ are plotted with various parameter sets in the FSUGold calculations. Though the isoscalar-isovector 
coupling is already included in the FSUGold, its strength is changed in FSUGw15 and FSUGw45 in order to manifest the importance of this coupling. Compared to results shown in Fig. 2, the role of the isoscalar-isovector coupling in the single-particle properties is similar in FSUGold, and here the nucleus ${ }^{292} 120$ is also doubly magic. Though the role of the isoscalar-isovector coupling is less prominent for the empirical shift between $\pi 3 s_{1 / 2}$ and $\pi 1 h_{9 / 2}$ than that in the NL3, it can favorably reduce the relative energy between these two levels. Similarly, we can establish correlations as in Fig. 4 for the FSUGold results, while here for brevity we neglect the display of the correlations. On the other hand, some distinctions of the single-particle spectrum are given with the FSUGold. For instance, an inversion of levels $\nu 1 k_{17 / 2}$ and $\nu 2 h_{11 / 2}$ is observed in the FSUGold calculations, while it does not take place in the NL3 calculations. The similar inversion also occurs between the $\nu 1 i_{11 / 2}$ and $\nu 3 p$ orbitals. Moreover, the shell gap at $\mathrm{N}=184$ is suppressed as compared to that with the NL3. On the contrary, the gap for N=198 in FSUGold well develops, and the isoscalarisovector coupling can further enhance the magnitude of this gap. This implies that $\mathrm{N}=198$ is a magic number with the FSUGold. With the moderate increase of the proton number, the $\mathrm{N}=198$ shell gap remains large. For instance, the size of the $\mathrm{N}=198$ shell gap in ${ }^{324} 126$ is even a little larger than that in ${ }^{318} 120$.

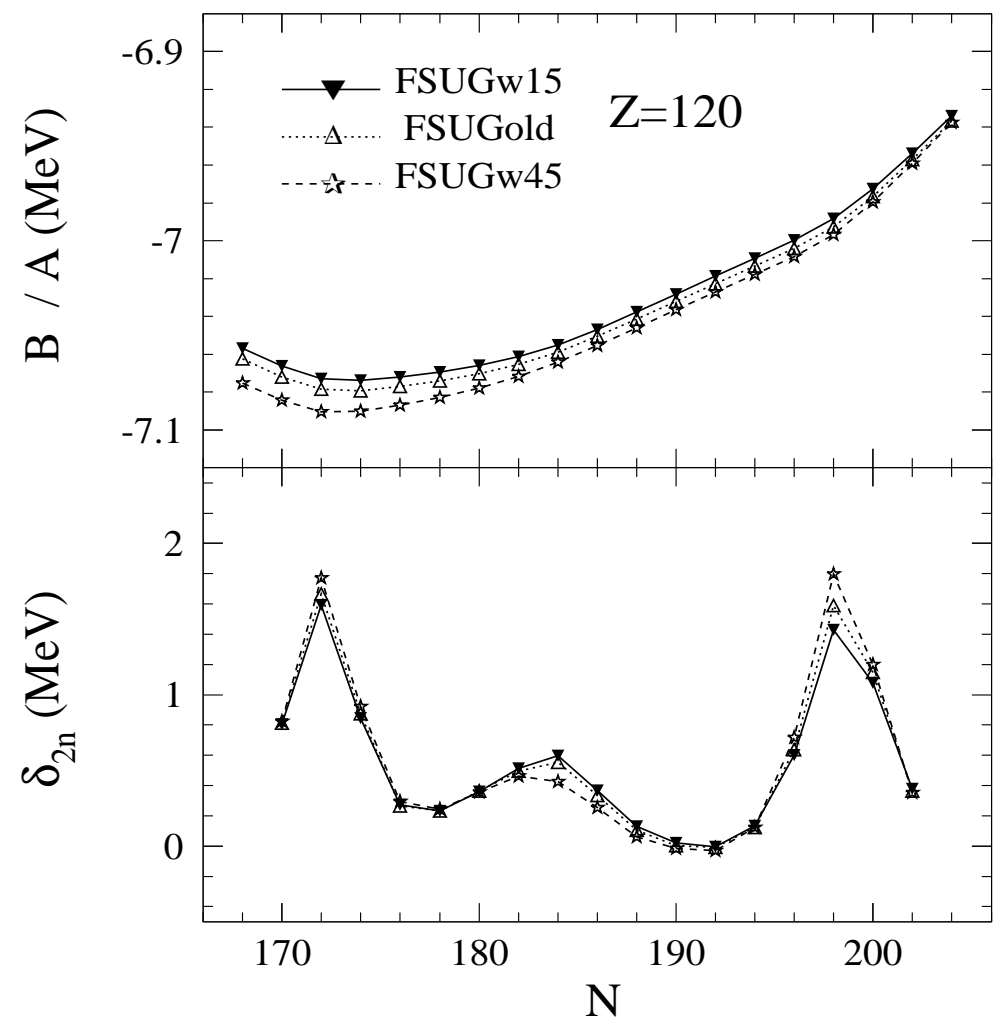

FIG. 8: The same as in Fig. 5 but for the FSUGold calculations. 
The $\mathrm{N}=198$ shell closure can consistently be observed using the $\delta_{2 n}$. Fig. 8 displays the binding energies and two-neutron gaps for the $\mathrm{Z}=120$ isotopes. As shown in the lower panel of Fig. 8, the two-neutron gap at $\mathrm{N}=198$ is clearly increased by softening the symmetry energy. Besides the large $\mathrm{N}=172$ gap, we see that the large $\mathrm{N}=198$ gap emerges. The situation of the shell closure at $\mathrm{N}=198$ in FSUGold differs from that in NL3. This distinction can be associated with different model constructions. For instance, the nonlinear self-interaction of the $\omega$ meson is included in FSUGold. Moreover, the compression modula of the NL3 and FSUGold differ by about $40 \mathrm{MeV}$. In general, the lower incompressibility in FSUGold allows more nucleons to be accommodated in the potential well. For the two-proton gap in FSUGold, it is less sensitive to differences in the symmetry energy than that in NL3, while the isotopic effect is similar to that in NL3. The increase of the neutron number suppresses the $\mathrm{Z}=120$ proton gap. The two-proton gap is 2.7 and $2.3 \mathrm{MeV}$ with $\mathrm{N}=172$ and 184, respectively. As compared to the NL3 results, the value of $\delta_{2 p}$ with the FSUGold turns out to be smaller. The peak disappears with $\mathrm{N}=198$, similar to that with the NL3.

To examine the consistency of the calculation, we plot in Fig. 9 the change in nucleon density distributions in the FSUGold calculations. It is shown that in ${ }^{318} 120$ the neutron density is enhanced in the central region. In presence of this central enhancement, the radial inhomogeneity appears to increase the sensitivity of the two-neutron gap to differences in the symmetry energy. For ${ }^{292} 120$, it was mentioned in Ref. [32] that the magnitude of the central depression increases with the decrease of the compression modulus. We note that the central depression in ${ }^{292} 120$ with the FSUGold is not more prominent than that with the NL3 as shown in Fig.3. Indeed, this can be attributed to the non-linear $\omega$-meson self-interaction in FSUGold that lowers the potential barrier. For ${ }^{304} 120$, as shown in the middle panel of Fig. 9, there exists an inhomogeneity of the modification added by the isoscalar-isovector coupling, and this is consistent with the moderate decrease of the two-neutron gap at $\mathrm{N}=184$ with the $\Lambda_{\mathrm{v}}$, as seen in the lower panel of Fig. 8 .

Next, we investigate the neutron skins in SHN. As it is known, the neutron skin thickness in heavy nuclei such as ${ }^{208} \mathrm{~Pb}$ is sensitive to differences in the symmetry energy. In SHN, the case is similar. In Fig. 10, we display neutron skin thicknesses in $\mathrm{Z}=120$ isotopes for various parameter sets in the NL3 and FSUGold calculations. In general, the sensitivity of the neutron skin thickness to differences in the symmetry energy can be well understood in the following way $[61,66,67]$. The pressure of pure neutron matter at saturation density is equal to the symmetry pressure. As the symmetry pressure decreases with the inclusion of the isoscalar-isovector coupling, the neutron skin thickness reduces in neutron-rich SHN. On the other hand, two interesting features in Fig. 10 are also observed. First, the neutron skin thicknesses in various SHN can roughly be separated into 


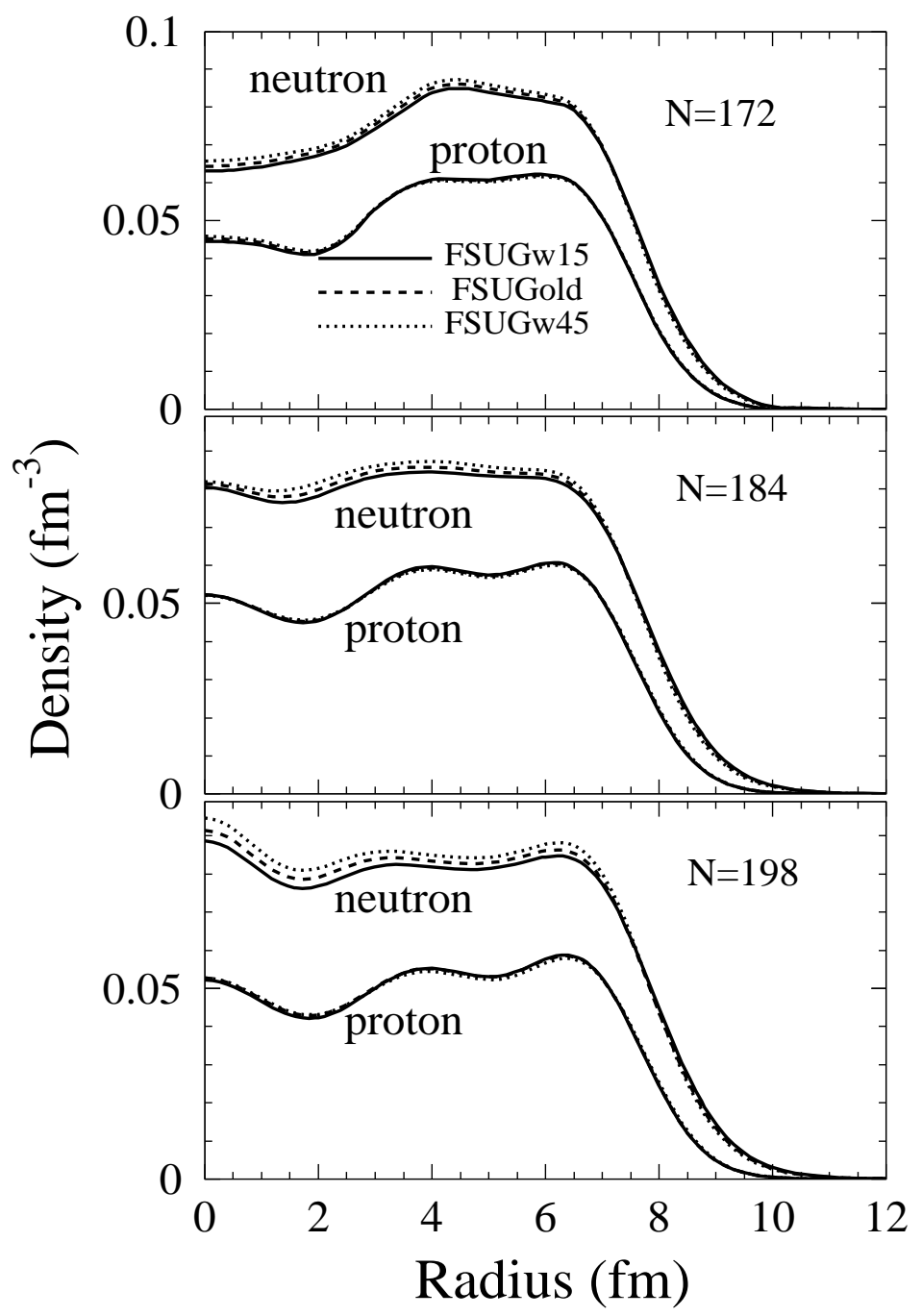

FIG. 9: Nucleon density distributions in the $\mathrm{Z}=120$ isotopes with various parameter sets in the FSUGold calculations.

two reaches according to the slope. The reach with the larger slope is associated with the large neutron gap at $\mathrm{N}=172$. With the addition of neutrons above this gap the neutron skin thickness thus increases clearly. As the occupation surpasses the much smaller gap at $\mathrm{N}=184$, the nuclear attraction from the interior gap can still appreciably restrain the extension of neutrons. This leads to a smaller slope at the larger isospin asymmetry. Second, we observe that the difference of the neutron skin thickness increases moderately at large isospin asymmetries. Because of the looser binding with the increase of the isospin asymmetry, it gives rise to an enhanced sensitivity to differences in the symmetry energy. Indeed, the correlation between the neutron skin and symmetry pressure in SHN deviates from a simple linear relation for heavy nuclei due to the more 


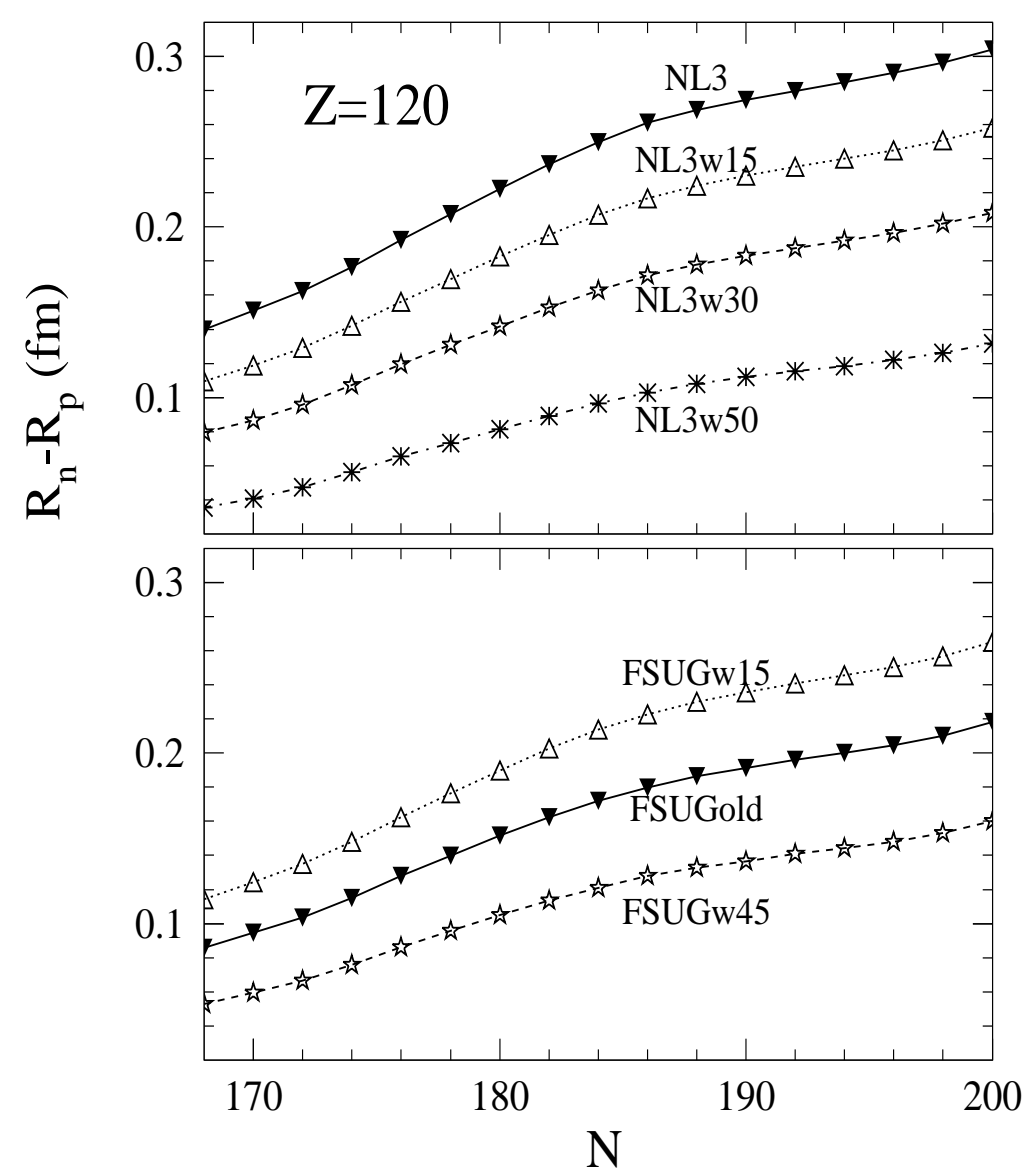

FIG. 10: Neutron skin thicknesses for SHN with various parameter sets in the NL3 (upper panel) and FSUGold (lower pannel) calculations.

extended neutron distribution given by a looser binding compared to that of heavy nuclei.

It is necessary to mention that the uncertainty of the neutron skin thickness is predominately from the changes in the neutron radius. Fig. 11 displays the charge radii of the $Z=120$ isotopes for various parameter sets in the NL3 and FSUGold calculations. It is shown that the change in charge (or, proton) radii is much smaller than the corresponding neutron skin thickness that is shown in Fig. 10. Moreover, for a considerable domain, it is seen that the charge radius of SHN can be well approximated as a constant. This is favorable, as one expects that the inclusion of the isoscalar-isovector coupling shouldn't compromise the success of models in reproducing a variety of ground-state properties. Recently, the precision measurement of the neutron radius of ${ }^{208} \mathrm{~Pb}$ has been proposed at the Jefferson laboratory via the parity-violating electron scattering on neutrons in ${ }^{208} \mathrm{~Pb}$ [68]. The measurement of the neutron radius that promises $1 \%$ accuracy will impose a strict constraint on the density dependence of the symmetry energy. Correspondingly, this also 


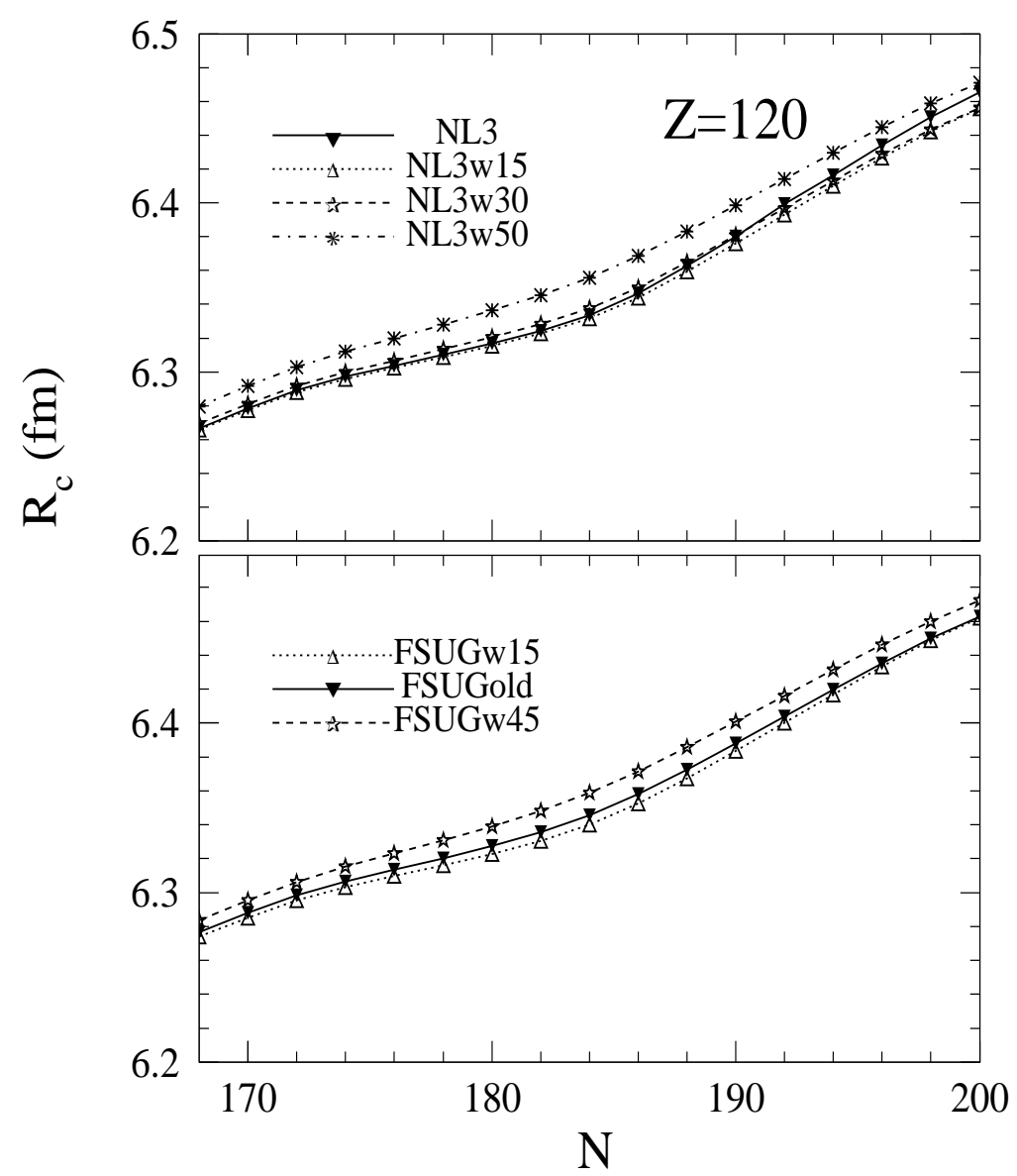

FIG. 11: The same as in Fig. 10 but for charge radii.

provides a significant constraint on the properties of SHN through the relationship that can be established for properties between SHN and ${ }^{208} \mathrm{~Pb}$.

At last, we mention a few weak points in this work. First, with the isoscalar-isovector coupling included or changed in the best-fit models, we fitted the corresponding parameters without using the best-fit procedure. Considering the important effect of the isoscalar-isovector coupling on the empirical shift, its inclusion seems necessary in the construction of the best-fit models for the study of SHN in future. Second, we note that the effect of the isoscalar-isovector coupling on the empirical shift of neutron levels is not as satisfactory as that of proton levels, though the prediction on the $\mathrm{N}=172$ shell closure in $\mathrm{SHN}$ is not much affected by this coupling. For a more detailed investigation of level shifts in future, it may be favorable to consider the coupling with the surface vibration modes $[69,70]$ and the influence of the relatively small Lorentz mass of nucleons in RMF models. Third, in this work we have used the simple BCS theory with phenomenological pairing gaps. For this point, we address it in some detail. It would be interesting to treat the pairing interaction in a 
dynamical way such as in the relativistic Hartree-Boguliubov (RHB) theory (for reviews, e.g., see Refs. [33, 53, 71]) for the open-shell SHN that are usually deformed, e.g., see Refs. [35-37], though the effect of pairing interactions on the shell closure is small. To our knowledge, a RHB model in the coordinate space is still not available for deformed nuclei, especially SHN [71], and in the RHB model the calculation for deformed nuclei is usually performed with the harmonic oscillator basis expansion. Due to the numerical complication, the RHB calculation in the deformed framework is still limited, see Refs. [34, 72] and references therein. In fact, most works treat the nucleon pairings in open-shell and deformed SHN using the BCS theory. It is interesting to note that the RMF model plus the BCS pairing works quite well for the open-shell nuclei except drip-line nuclei [73, 74], since for most isotopes the nucleon occupation number in the continuum is just moderate. For most open-shell SHN, we find that the occupation in the continuum is also comparatively small. In this sense, the results obtained for most open-shell SHN with the RMF model plus the BCS pairing are comparable to those obtained with the RHB model. Recently, a separable pairing force was proposed for the RHB model with considerable reduction of the computing time [72], and it would be hopefully developed to study properties of open-shell SHN.

\section{SUMMARY}

In summary, we have investigated the dependence of the ground-state properties of spherical SHN on the density dependence of the symmetry energy within RMF models. The various density dependences of the symmetry energy are simulated by changing the strength of the isoscalarisovector coupling in RMF models (the NL3 and FSUGold). It is found that the isoscalar-isovector coupling produces an important effect on the empirical shift of spherical orbitals in SHN. Especially,

the empirical shift between the $\pi 3 s_{1 / 2}$ and $\pi 1 h_{9 / 2}$ in NL3 is nicely reproduced by including the isoscalar-isovector coupling. This provides a favorable support for the $\mathrm{Z}=120$ shell closure. In addition, the isoscalar-isovector coupling can produce a small but favorable effect on the $\mathrm{N}=172$ shell closure. With both models NL3 and FSUGold that differ in the compression modulus, the double shell closure is predicted in ${ }_{172}^{292} 120$. The shell closure is also investigated in more $\mathrm{Z}=120$ isotopes. We have discussed the association of the central depression or enhancement with the effect of the softening of the symmetry energy in SHN. Compared to the moderate modification to single-particle energies and shell gaps, significant reduction of the neutron skin thickness in SHN is expectedly obtained by softening the symmetry energy. Moreover, the proton radius is little changed, similar to the situation in ${ }^{208} \mathrm{~Pb}$. 


\section{Acknowledgement}

The work was supported in part by the National Natural Science Foundation of China under

Grant No. 10975033, the China Jiangsu Provincial Natural Science Foundation under Grant

No.BK2009261, the Knowledge Innovation Project of the Chinese Academy of Sciences under

Grant No. KJXC3-SYW-N2, and the China Major State Basic Research Development Program under Contract No. 2007CB815004.

[1] S. Hofmann, Rep. Prog. Phys. 61, 639 (1998).

[2] S. Hofmann and G. Münzenberg, Rev. Mod. Phys. 72, 733 (2000).

[3] P. A. Wilk, K. E. Gregorich, A. Türler, et.al., Phys. Rev. Lett. 85, 2697(2000).

[4] Yu. Ts. Oganessian, V. K. Utyonkov, Yu. V. Lobanov, et.al., Phys. Rev. C 69, 021601(R) (2004).

[5] Yu. Ts. Oganessian, V. K. Utyonkov, Yu. V. Lobanov, et.al., Phys. Rev. C 70, 064609 (2004).

[6] K. Morita, K. Morimoto, D. Kaji, et. al., Eur. Phys. J. A 21, 257 (2004).

[7] Z. G. Gan, J. S. Guo, X. L. Wu, et.al., Eur. Phys. J. A 20, 385 (2004).

[8] J. Dvorak, W. Brüchle, M. Chelnokov, et.al., Phys. Rev. Lett. 97, 242501 (2006).

[9] Yu. Ts. Oganessian, V. K. Utyonkov, Yu. V. Lobanov, et.al., Phys. Rev. C 74, 044602 (2006).

[10] Yu. Ts. Oganessian, J. Phys. G: Nucl. Part. Phys. 34, R165 (2007).

[11] Yu. Ts. Oganessian, V. K. Utyonkov, Yu. V. Lobanov, et.al., Phys. Rev. C 76, 011601(R) (2007).

[12] A. Sobiczewski, and K. Pomorski, Prog. Part. Nucl. Phys. 58, 292 (2007).

[13] S. L. Nelson, K. E. Gregorich, I. Dragojevic, et.al., Phys. Rev. Lett. 100, 022501 (2008).

[14] M. Morjean, D. Jacquet, J. L. Charvet, et.al., Phys. Rev. Lett. 101, 072701 (2008).

[15] P. Möller and J. R. Nix, Nucl. Phys. A 549, 84 (1992); J. Phys. G 20, 1681 (1994).

[16] M. Bender, K. Rutz, P.-G. Reinhard, et.al., Phys. Rev. C 60, 034304 (1999).

[17] K. Rutz, M. Bender, T. Bürvenich, et.al., Phys. Rev. C 56, 238 (1997).

[18] J. Decharge, J.-F. Berger, M. Girod, and K. Dietrich, Nucl. Phys. A 716, 55 (2003).

[19] S. Ćwiok, J. Dobaczewski, P.-H. Heenen, et.al., Nucl. Phys. A 611, 211 (1996).

[20] W. Zhang, J. Meng, S.Q. Zhang, et.al., Nucl. Phys. A 753, 106 (2005).

[21] J. M. Lattimer and M. Prakash, Phys. Rep. 333, 121 (2000); Science 304, 536 (2004).

[22] C. J. Horowitz, J. Piekarewicz, Phys. Rev. Lett. 86, 5647 (2001).

[23] A. W. Steiner, M. Prakash, J. M. Lattimer and P. J. Ellis, Phys. Rep. 411, 325 (2005).

[24] B.G.Todd, J.Piekarewicz, Phys. Rev. C 67, 044317 (2003).

[25] W. Z. Jiang, Y. L. Zhao, Phys. Lett. B 617, 33 (2005).

[26] B. A. Li, Phys. Rev. Lett. 85, 4221 (2000); ibid. 88, 192701 (2002).

[27] B. A. Li, L. W. Chen, and C. M. Ko, Phys. Rep. 464, 113 (2008).

[28] L. W. Chen, C. M. Ko, B. A. Li, Phys. Rev. Lett. 94, 032701 (2005).

[29] B. A. Li, L. W. Chen, Phys. Rev. C72, 064611 (2005).

[30] M. B. Tsang et.al. Phys. Rev. Lett. 92, 062701 (2004).

[31] J. Decharge, J.-F. Berger, K. Dietrich, and M. S. Weiss, Phys. Lett. B 451, 275 (1999).

[32] A. V. Afanasjev, and S. Frauendorf, Phys. Rev. C 71, 024308 (2005).

[33] A. V. Afanasjev, T. L. Khoo, S. Frauendorf, et.al., Phys. Rev. C 67, 024309 (2003).

[34] A. V. Afanasjev, nucl-th/0612095

[35] Z. Ren and H. Toki, Nucl. Phys. A 689, 691 (2001).

[36] Z. Ren, F. Tai, and D.-H. Chen, Phys. Rev. C 66, 064306 (2002).

[37] Z. Ren, Phys. Rev. C 65, 051304 (2002).

[38] Z. Ren, D. H. Chen, F. Tai, et.al., Phys. Rev. C 67, 064302 (2003).

[39] S. Typel and B. A. Brown, Phys. Rev. C 67, 034313 (2003).

[40] F. R. Xu, E. G. Zhao, R. Wyss, and P. M. Walker, Phys. Rev. Lett. 92, 252501, (2004).

[41] T. Sil, S. K. Patra, B. K. Sharma, et.al., Phys. Rev. C 69, 044315 (2004).

[42] A. Baran, Z. Lojewski, K. Sieja, and M. Kowal, Phys. Rev. C 72, 044310 (2005).

[43] M. M. Sharma and A. R. Farhan, and G. Münzenberg, Phys. Rev. C 71, 054310 (2005).

[44] J. C. Pei, F. R. Xu, Z. J. Lin, and E. G. Zhao, Phys. Rev. C 76, 044326 (2007).

[45] T. Dong, and Z. Ren, Phys. Rev. C 77, 064310 (2008).

[46] C. J. Horowitz, J. Piekarewicz, Phys. Rev. C 64, 062802(R) (2001).

[47] C. J. Horowitz, J. Piekarewicz, Phys. Rev. C66, 055803 (2002).

[48] B. G. Todd-Rutel, J. Piekarewicz, Phys. Rev. Lett. 95, 122501 (2005). 
[49] G. Shen, J. Li, G. C. Hillhouse, and J. Meng, Phys. Rev. C 71, 015802 (2005).

[50] W. Z. Jiang, Phys. Lett. B 642, 28 (2006).

[51] B. D. Serot, J. D. Walecka, Adv. Nucl. Phys. 16, 1 (1986); Int. J. Mod. Phys. E 6, 515 (1997).

[52] Y. K. Gambhir, P. Ring and A. Thimet, Ann. Phys. (N.Y.) 198, 132 (1990).

[53] P. Ring, Prog. Part. Nucl. Phys. 37, 193 (1996).

[54] P. Möller and J. R. Nix, Nucl. Phys. A 536, 20 (1992).

[55] G. A. Lalazissis, J.König, P.Ring, Phys. Rev. C 55, 540 (1997).

[56] I. Ahmad, F.T. Porter, M.S. Freedman, et.al., Phys. Rev. C 3, 390 (1971).

[57] I. Ahmad, R.K. Sjoblom, and P.R. Fields, Phys. Rev. C 14, 218 (1976).

[58] I. Ahmad, R.R. Chasman, A.M. Friedman, and S.W. Yates, Phys. Lett. B 251, 338 (1990).

[59] I. Ahmad, M.P. Carpenter, R.R. Chasman, J.P. Greene, et.al., Phys. Rev. C 62, 064302 (2000).

[60] B. A Li, C. M. Ko, and W. Bauer, Int. J. Mod. Phys. E 7, 147 (1998).

[61] R. J. Furnstahl, Nucl. Phys. A 706, 85 (2002).

[62] J. Boguta and A. R. Bodmer, Nucl. Phys. A 292, 413 (1977).

[63] J. Boguta and S. A. Moszkowski, Nucl. Phys. A 403,445 (1983).

[64] G. A. Lalazissis, M.M. Sharma, P. Ring, and Y.K. Gambhir, Nucl. Phys. A 608, 202 (1996).

[65] M. Rashdan, Phys. Rev. C 63, 044303 (2001).

[66] B. A. Brown, Phys. Rev. Lett. 85, 5296 (2000).

[67] L. W. Chen, C. M. Ko and B. A. Li, Phys. Rev. C 72, 064309 (2005).

[68] R.Michaels, P.A.Souder, G.M.Urciuoli, spokespersons, Jefferson Laboratory Experiment E-00-003.

[69] C. Mahaux, P. F. Bortignon, R.A. Broglia, and C.H. Dasso, Phys. Rep. 120, 1 (1985).

[70] D. Vretenar, T. Niksic, and P. Ring, Phys. Rev. C 65, 024321 (2002).

[71] J. Meng, H. Toki, S. G. Zhou, S. Q. Zhang, W.H. Long, L.S. Geng, Prog. Part. Nucl. Phys. 57, 470 (2006).

[72] Y. Tian, Z. Y. Ma and P. Ring, Phys. Rev. C 80, 024313 (2009).

[73] J.Q. Li, Z.Y. Ma, B.Q. Chen and Y. Zhou, Phys. Rev. C 65, 064305 (2002).

[74] W.Z. Jiang, Z.Z. Ren, T.T. Wang, Y.L. Zhao, and Z.Y. Zhu, Eur. Phys. J. A 25, 29 (2005). 